TAO, Vol. 12, No. 4, 615-634, December 2001

\title{
An Estimation of Typhoon Intensity and the Prediction of Its Track by Using MSU Data
}

\author{
Gin-Rong Liu ${ }^{1,2, *}$, Tsung-Hua Kuo ${ }^{1,3}$, Pai-Ying Leu ${ }^{2}$, Tang-Huang Lin ${ }^{1,3}$, and Chung-Chin Liu ${ }^{4}$
}

(Manuscript received 31 January 2000, in final form 8 October 2001)

\begin{abstract}
This study uses both airborne and NOAA (National Oceanic and Atmospheric Administration) MSU (Microwave Sounding Unit) observational data, covering the Taiwan area during the 1990 TATEX (Taiwan Area Typhoon Experiment) period to develop a typhoon intensity estimation model. Although some previous studies have shown that the temperature difference of the typhoon center and its environment estimated by the MSU at $250-\mathrm{mb}$ level has a positive relationship with the maximum wind speed, the results in this paper show that they are not as satisfactory as expected. When using only the $250-\mathrm{mb}$ temperature anomaly, the value of the correlation is roughly about 0.6, and the RMSE (Root Mean Square) being 19kts. According to the sensor readings of the weighting function, this outcome suggests that the maximum typhoon wind speed is strongly related to the brightness temperature, which is contributed throughout each layer of the typhoon. Thus, the wind speed information is not complete when only the upper levels of the temperature information are considered. Because the weighting function of the third MSU channel extends downward all the way to the $700-\mathrm{mb}$ level, additional information can be extracted for the retrieval of the intensity of a typhoon. The result shows an improvement in predicting the typhoon intensity accuracy with the correlation and RMSE equaling 0.7 and $16.5 \mathrm{kts}$, respectively when both the 250 and $700-\mathrm{mb}$ temperature anomalies are considered simultaneously.

We also used a 700-mb cold-core location in predicting the path a typhoon follows. The results showed a value of $0.9 \mathrm{R}$-squared and an RMSE of 13.1 degrees in angle. Generally speaking, the results were rather impressive and pave the way for further exploration of the method's potential application in monitoring a typhoon's track.
\end{abstract}

\footnotetext{
${ }^{1}$ Center for Space and Remote Sensing Research, National Central University, Chung-Li, Taiwan

${ }^{2}$ Institute of Atmospheric Physics, National Central University, Chung-Li, Taiwan

3 Institute of Space Science, National Central University, Chung-Li, Taiwan

${ }^{4}$ Air Force Aeronautical and Technical School, Gang-Shan, Taiwan

* Corresponding author address: Prof. Gin-Rong Liu, Center for Space and Remote Sensing Research, National Central University, Chung-Li, Taiwan 320; E-mail: grliu@csrsr.ncu.edu.tw
} 


\section{(Key words: TATEX, MSU, Typhoon intensity, Remote Sensing)}

\section{INTRODUCTION}

For a very long time, typhoon monitoring mostly relied on airborne instruments to observe a typhoon's parameters. Although these methods can obtain accurate data, they require extensive investment in manpower and equipment that is not considered economical, especially over the open ocean. Satellite observations serve as an excellent alternative in providing this information because of its wide coverage and sharp temporal resolution.

Since microwave sensors were put into earth orbits in the last two decades, much insight of tropical cyclone structure and behavior has been gained by using microwave data. For example, Allison et al. (1974) used microwave data from Nimbus-5 to estimate the rainfall of tropical cyclones. Kidder et al. (1980) employed together the static equilibrium assumption, the microwave radiative equation (RTE) and the gradient wind equation to estimate a typhoon's intensity. Grody (1983) proposed that the MSU data of Channels $1(50.31 \mathrm{GHz})$ and 2 (53. $73 \mathrm{GHz}$ ), having a resolution of $110 \mathrm{~km}$ in-nadir, could be employed to find the rainfall area within a typhoon. They took advantage of the fact that the microwave brightness temperatures were significantly affected by rainfall scattering, which was verified by concurrent radar observations. In addition, they also suggesied that Channel $3(54.96 \mathrm{GHz})$ could be used to observe the size and intensity of a typhoon's warm-core because it was little affected by rainfall. Velden and Smith (1983) suggested that the variation of the warm-core might be an indication of a typhoon intensifying or weakening. Based on the previous studies conducted by other research teams, they also used MSU data to establish a regression relationship between the 250-mb temperature anomalies, the surface pressure difference and the maximum wind speed in Northern Atlantic hurricanes. Further on, Velden et al. (1991) used three years of MSU satellite data to establish a typhoon intensity regression, but found that the accuracy was not good enough. Their analysis revealed that the height of the warm-core changed along with different latitudes, typhoon intensities, or environmental parameters. Moreover, the vertical temperature structure could not be accurately represented by a single $250-\mathrm{mb}$ channel weighting function.

Nevertheless, satellite microwave data can be used to observe many typhoon parameters, such as rainfall rates, total water vapor content, wind speed, latent heat flux, and so forth (Chang et al. 1993; Petty et al. 1994; Rodgers et al. 1993; Huang et al. 1992). These estimations can be further used to assess a typhoon's intensity or plot its path.

The aim of this current study is to analyze microwave data and establish a model in estimating a typhoon's intensity by using data acquired from the MSU and aircraft instruments taken during the Taiwan Area Typhoon Experiment (TATEX). In addition, we also tried to predict a typhoon's path of movement 6 hours in advance.

\section{THEORY}

\subsection{Microwave Radiative Transfer Equation}

In the microwave region, the radiation intensity is proportional to the absolute tempera- 
ture $(\mathrm{K})$. Therefore, the microwave radiative transfer equation (RTE) can be written as

$$
\begin{aligned}
& T_{B}(\bar{v})=\mathfrak{I}_{\bar{v}}\left(\frac{\tau_{1}}{\mu}\right)\left[\varepsilon_{\bar{v}_{s}} T_{s}+\left(1-\varepsilon_{\bar{v}_{s}}\right) \int_{0}^{\tau_{1}} T\left(\tau^{\prime}\right) \frac{\partial \mathcal{J}_{\bar{v}}^{d}\left(\tau_{1}-\tau^{\prime}\right)}{\partial \tau^{\prime}} d \tau^{\prime}\right] \\
& -\int_{0}^{\tau_{1}} T\left(\tau^{\prime}\right) \frac{\partial \mathfrak{I}_{\bar{v}}\left(\tau^{\prime} / \mu\right)}{\partial \tau^{\prime}} d \tau^{\prime},
\end{aligned}
$$

where $\mu=\cos \theta$, and $\theta$ is the satellite observation angle, $\mathfrak{I}_{\bar{\nu}}$ is the upwelling transmittance, $\mathfrak{J}_{\bar{v}}^{d}$ is the downwelling transmittance, $\tau$ is the optical depth, $T_{s}$ is the surface temperature, and $\varepsilon_{v_{s}}$ is the surface emissivity. As we see from the equation, $\mu=1$ when the satellite is in nadir. If the horizontal axis is designated to be in pressure coordinates, the microwave RTE can be rewritten into the following:

$$
T_{B}(\bar{v})=\varepsilon_{\bar{v}_{s}} T_{s} \mathfrak{J}_{\overline{v_{s}}}+\int_{0}^{p} k(\bar{v}, P) T(P) \frac{d P}{P},
$$

where $k(\bar{v}, P)$,is the temperature weighting function.

\subsection{Relationship between Temperature Anomalies and Maximum Wind Speed}

Kidder (1980) argued that the evolution of tropical cyclones could be treated as a series of quasi-steady states within certain time scaling. By relating this assumption to the RTE in the microwave region, Kidder further derived a model for estimating the maximum wind speed by using the brightness temperature anomalies and pressure differences within the typhoon center.

The brightness temperature observed by the satellite sensor is contributed by a temperature reading in every individual atmospheric layer, which starts from the surface and ends at the very top of the atmosphere (TOA). If channels that are insensitive to the surface contribution are used, $\mathrm{Eq}(2)$ can be simplified as:

$$
T_{B}(r)=\int_{0}^{H} K_{\lambda}(Z) T(r, Z) d Z,
$$

where $r$ is defined as the distance to the typhoon's center, and $K_{\lambda}(Z)$ is the weighting function. We then separate the observed temperature, $T(r, Z)$, into two parts---the environmental temperature, $T_{E}(Z)$, and the temperature anomaly, $T^{\prime}(r, Z)$,

$$
T(r, Z)=T_{E}(Z)+T^{\prime}(r, Z)=T_{E}(Z)+\alpha(r) T^{*}(Z),
$$

where $\alpha(r)$ is the intensity parameter and the appearance of $\alpha(r)$ is only for the convenience of mathematical derivation. $T *(Z)$ is the ideal temperature anomaly profile (Velden 1989). Thus, Eq (3) can now be expanded into the following:

$$
\begin{aligned}
& T_{B}(r)=\int_{0}^{H} K_{\lambda}(Z)\left[T_{E}(Z)+\alpha(r) T^{*}(Z)\right] d Z \\
& =T_{B_{E}}+\alpha(r) \int_{0}^{H} K_{\lambda}(Z) T^{*}(Z) d Z,
\end{aligned}
$$


where $T_{B_{E}}$ is the environmental brightness temperature. In the static equilibrium assumption, by substituting $\mathrm{Eq}(5)$ into the static equilibrium equation, the following relationships can be derived:

$$
\begin{aligned}
& \frac{\Delta \ln P_{S}}{\Delta T_{S}}=-\frac{g}{R} \int_{0}^{H_{r}} \frac{T^{*}(Z)}{T_{E}^{2}(Z)} d Z\left[\int_{0}^{H} K_{\lambda}(Z) T^{*}(Z) d Z\right]^{-1} \equiv-A, \\
& \Delta \ln P_{S}=\ln P_{S}(r)-\ln P_{S_{E}} ; \\
& \Delta T_{S}=T_{B}(r)-T_{B_{E}},
\end{aligned}
$$

where $P_{S}$ and $T_{S}$ are the surface pressure and temperature, respectively. $H_{r}$ is the height where the atmosphere is not influenced by the cyclone. By combining together the typhoon's pressure at the center $P_{C}$, the typhoon's brightness temperature at the center $T_{B_{\mathrm{c}}}$, the environmental pressure $P_{E}$, and finally the environmental brightness temperature $T_{B_{E}}, R$ is the ideal gas parameter, $A$ is a ratio factor, we obtain the relationship:

$$
\ln \frac{P_{C}}{P_{E}}=-A\left(T_{B_{C}}-T_{B_{E}}\right) .
$$

Eq (7) shows that a linear relationship exists between the brightness temperature anomaly and the pressure difference between the typhoon's center and the environment.

By considering the gradient wind equation:

$$
\frac{V^{2}}{r}+f V=R T \frac{\partial \ln P}{\partial r} .
$$

We may infer that the radial temperature gradients below the $850-\mathrm{mb}$ height will drop to nearly zero because the maximum wind speed usually occurs at this height. Thus:

$$
\frac{\partial}{\partial z}\left(\frac{\partial \ln P}{\partial r}\right)=\frac{\partial}{\partial r}\left(\frac{\partial \ln P}{\partial z}\right)=\frac{\partial}{\partial r}\left(-\frac{g}{R T}\right) \approx 0 .
$$

This means that the radial pressure gradient $\partial \ln P / \partial r$ in Eq (9) can be replaced with the surface radial gradient $\partial \ln P_{S} / \partial r$. Moreover, we see that $\Delta \ln P_{S}$ has a linear connection with $\Delta T_{B}$. When considering that $P$ is only a function of $r$, the variation in $\ln P_{S}$ versus the change in $r$ equals the variation in $T_{B}$ versus the change in $r$. This allows the radial pressure gradient to be replaced by the brightness temperature gradient, which is not influenced by the surface emissivity. Basically, the typhoon cases analyzed in this study contained few super typhoons and the weighting function at $850-\mathrm{mb}$ is rather similar to the weighting function near the surface. Therefore, the estimation error does not seem to be very large. In addition, the gradient wind equation can be rewritten as:

$$
\frac{V^{2}}{r}+f V=-A R T \frac{\partial T_{B}}{\partial r},
$$

where $f$ is the Coriolis parameter. Eq (10) shows the relationship between the typhoon wind speed and the brightness temperature. If the satellite-borne microwave sensors have a sharp spatial resolution, the maximum wind speed can be estimated based upon these two assumptions. 
Unfortunately, the MSU is currently unable to provide such resolution. Therefore, Velden et al.(1991) used a 250-mb temperature anomaly, $\Delta T_{250}$, to establish the estimation algorithm for assessing the typhoon maximum wind speed $V_{\max }$. They claimed that the reason why the 250-mb layer contained the strongest warm-core structure was that it corresponded to the weighting function peak in the third MSU channel.

\section{DATA PRE-PROCESSING}

The year 1990 was considered International Typhoon Year, as well as being the last year for the Western Pacific typhoon aircraft observation to be officiated by the U.S. Joint Typhoon Warning Center at Guam. Taking advantage of the aircraft observations, TATEX was also simultaneously executed. Therefore, the typhoon cases of 1990 were chosen for analysis so that we could make use of the aircraft observations in validating and confirming our estimation results. The aircraft data included observations of five different typhoons (Winona, Yancy, Zola, Abe and Becky). The status of the storm's center and the velocity of the maximum wind speed were reported every six hours. We also used data from the MSU that was within the same time frame of the aircraft flyby. The data had coverage of more than seven latitudes. Nine MSU images that satisfied these conditions were chosen during the 8-day Winona life cycle. Similarly, 9 MSU images were selected from the 14-day long Yancy, 4 from the 9-day long Zola, 9 from the 9-day long Abe, and 6 from the 11-day long Becky, respectively. In total, $37 \mathrm{MSU}$ images were chosen.

Indeed, the poor resolution of the MSU data presents a great problem for application. Therefore, the temperature fields used in our analysis were retrieved by ITPP (International TOVS Processing Package) where both MSU and HIRS (High Resolution Infrared Radiation Sounder) data were inputted. Generally, HIRS data were used in clear pixels. For cloudy pixels, HIRS data were used in higher levels, (above cloud layer) and the MSU data were used for lower levels (below cloud layer). Finally the estimated temperature fields from ITPP were resampled into HIRS resolution, $18.5 \mathrm{~km}$ in-nadir. Hence, the temperature fields used in this study will have a somewhat better resolution than the MSU. For example, the temperature fields at 250-mb were usually located above the cloud layer rendering their spatial resolution to be expressed in terms of HIRS instead of the other. One other reason for using MSU in this study was that the results could be verified by routine aircraft observations, which were stopped after 1987. Although the AMSU has a much better resolution, they have only been available in recent years.

In addition, even for some analyzing cases $(850-\mathrm{mb}$ or part $700-\mathrm{mb}$ case) that are below the cloud, the gradient fields can still be correctly determined in most situations because they are usually computed in a $300 \mathrm{~km}$ distance, which still owns a larger resolution than the 110 $\mathrm{km}$ of the MSU. We do agree that necessary corrections should be done to improve the MSU applications' accuracy. In fact, some standard correction procedures have been established inside the ITPP.

\subsection{Angle Correction}

Owing to the different scanning angles, especially for the large view angle of microwave 
sensors, a limb darkening effect was observed in the scanned images. This effect could induce an overestimation of the weighting function peak height to 30-mb (Grody 1983). Therefore, angle correction work was necessary in order to obtain accurate typhoon temperature field distributions.

The sounding and satellite data we collected from around East Asia were used to correct the angle effect. Only image pixels near the sounding station that was no further than 18 kilometers away were used. After analyzing the relationships between the brightness temperature values, the scanning angles, and the brightness temperatures simulated by the sounding data, the non-linear limb darkening effect was overcome. However, because of the lack of surface emissivity data, MSU Channel 1 could not be corrected in this study.

\subsection{Rainfall Effect Masking}

By comparing typhoon observations, soundings, and radar data, it was found that a typhoon's strongest rainfall area can be identified with the low brightness temperature pixels of MSU Channel 2 (Grody 1983). Because Channel 2 is sensitive to rainfall, it is therefore weakened by the strong precipitation in the middle troposphere. This weakening effect is basically proportional to the rainfall intensity. Therefore, the rainfall effect correction should be done in advance before Channels 2,3 , and $4(57.95 \mathrm{GHz})$ are used to retrieve the temperature profiles. Yet, even by doing so, we were still unable to completely eliminate the powerful effect the rainfall produced on the brightness temperature in some pixels. Therefore, in order to ensure a reliable estimated result, the affected pixels were not used.

The angle-corrected data of MSU Channel 2 were used to identify the rainfall area in our study. In general, areas having lower brightness temperatures indicated stronger precipitation. A rainfall threshold value was chosen to distinguish the strong rainfall MSU pixels from the others.

\section{TEMPERATURE FIELD ANALSIS}

After first conducting the required processing of the $37 \mathrm{MSU}$ images, ITPP model was employed to retrieve the temperature field. In addition, the objective analysis method was utilized in obtaining the brightness temperature fields. The $250-\mathrm{mb}$ and $700-\mathrm{mb}$ temperature fields were then used to analyze the typhoon. Figures. 1 and 2 are the $250-\mathrm{mb}$ and $700-\mathrm{mb}$ temperature fields respectively for Typhoon Yancy. From the temperature field figures, it can be seen that the typhoon's warm-core is located at the upper convective layer, mainly at the 250-mb layer. This result coincides with our previous discussions in this study. Meanwhile, as cold temperature anomalies can exist outside a typhoon's eye below the $600-\mathrm{mb}$ layer, the $250-\mathrm{mb}$ and $700-\mathrm{mb}$ fields were chosen for data analysis in gauging the typhoon's intensity.

\subsection{0-mb Temperature Field}

The analysis revealed that the maximum wind speed in the typhoon could reach $70 \mathrm{kts}$, which is shown in Fig. la. Significant increases were observed in the last two days, where a 
8/16 22:57-23:05 250mo(s.p)

8/19 06:01-06:09 250mb(c.p)
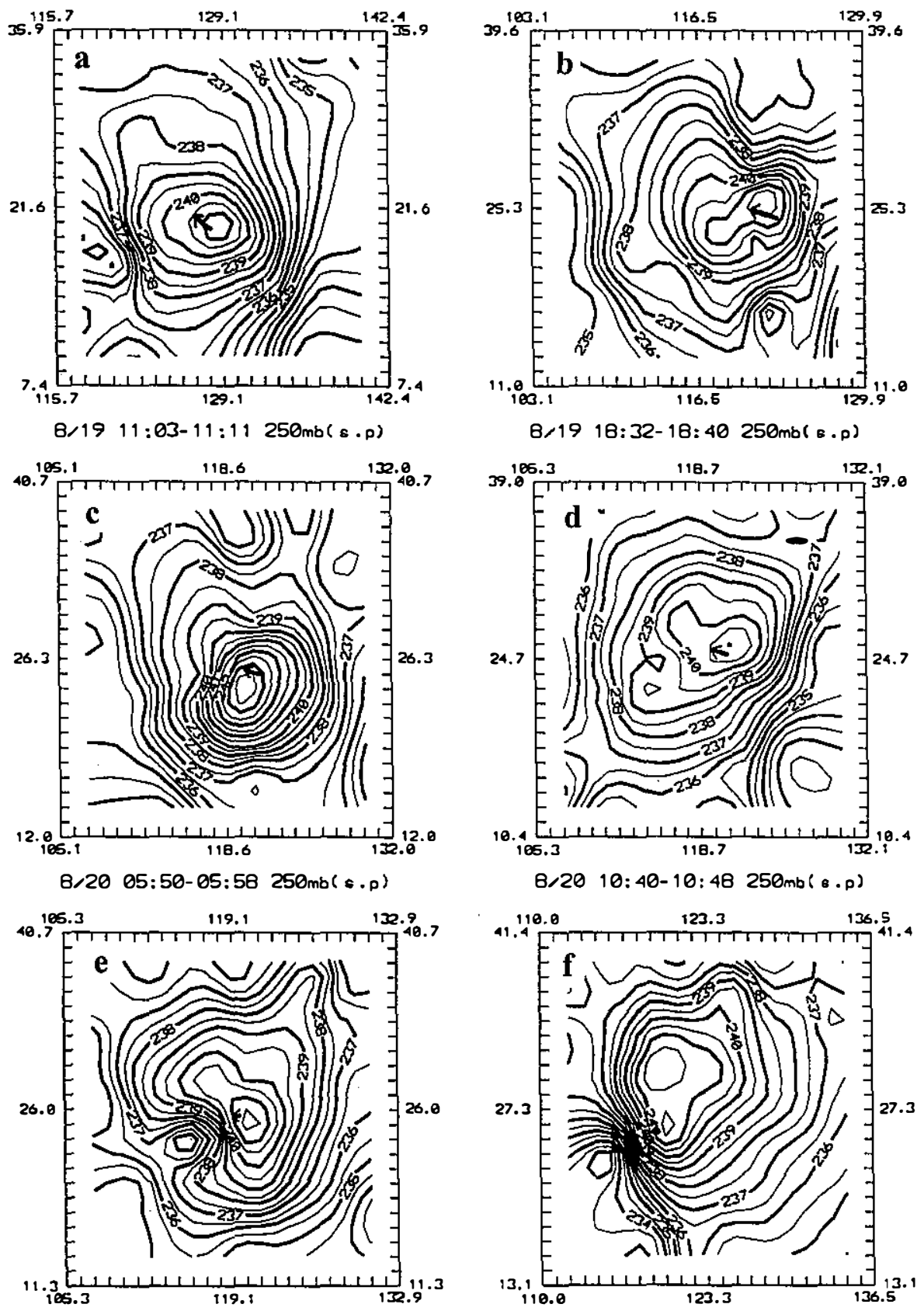

Fig. 1. The 250-mb temperature fields for Typhoon Yancy, (a) 22:57 GMT August 16, 1990, (b) 06:01 GMT August 19, (c) 11:03 GMT August 19, (e) 05:50 GMT August 20, (f) 10:40 GMT August 20, (g) 18:22 GMT August 20, (h) 18:10 GMT August 21, and (i) 11:35 GMT August 22. 

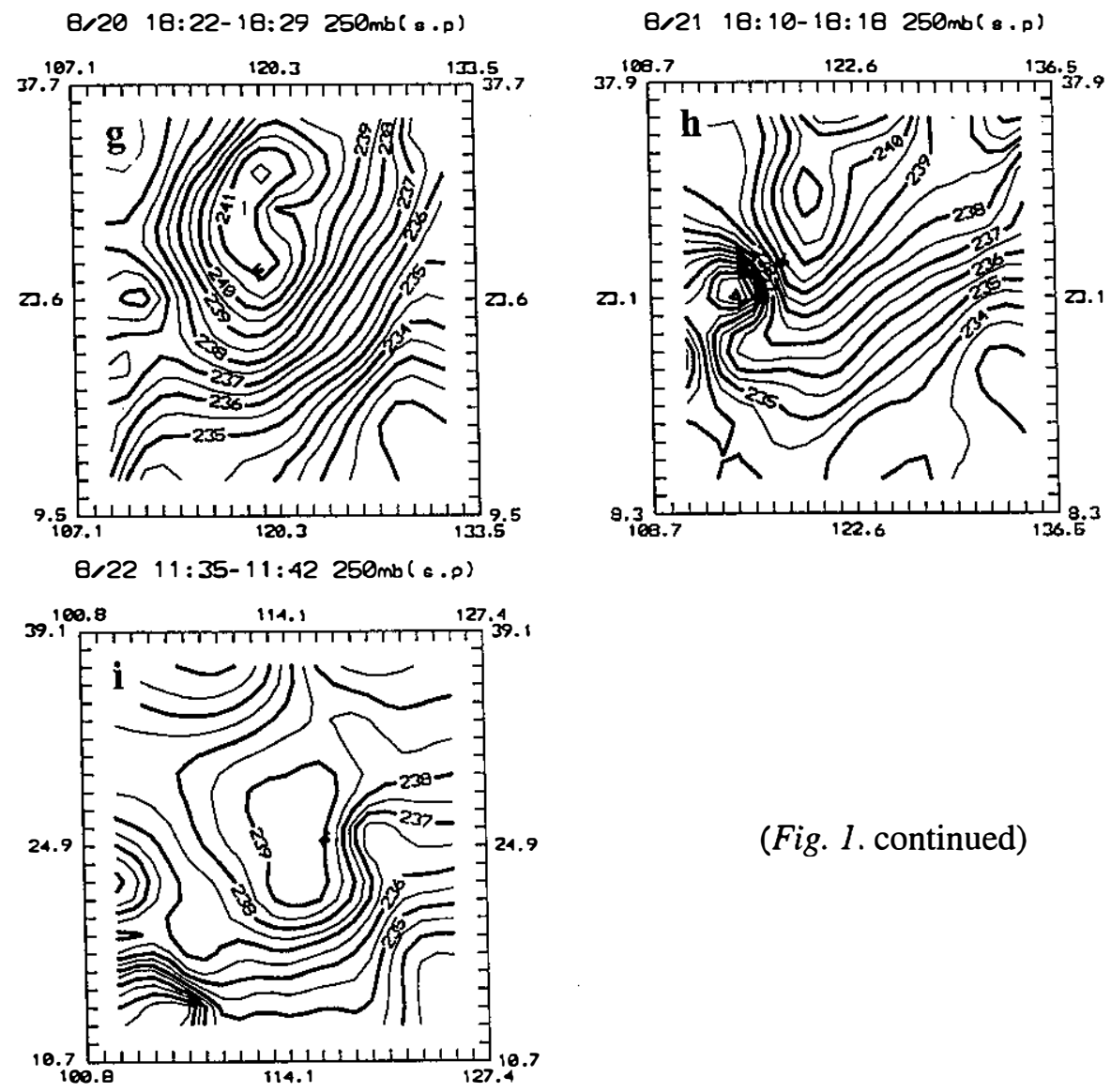

(Fig. 1. continued)

maximum was attained on August 18. However, the typhoon was not located in the coverage area of the MSU at the time, making it unable to make comparisons. Meanwhile, in Fig. 1b, we see that the typhoon's maximum wind speed decreased from 85kts at 2300UTC on Aug 16 to below $55 \mathrm{kts}$ at 0600 UTC on Aug17. The warm-core separated slowly from the typhoon center as Yancy moved into Mainland China where its intensity dropped below 55kts (Figs. 1e to 1 i).

It is important to note that data from MSU Channel 1 was not directly used in the ITPP package for temperature retrieval, but used instead to retrieve surface emissivity. Therefore, if the angle correction is not performed accurately, the emissivity may be underestimated, inducing overestimations in the temperature field. When a typhoon approaches land, the warmest region in the MSU images will appear over the land and not at the supposedly typhoon's center. This anomaly can be easily observed, especially in some weak typhoons, such as Typhoon Winona.

Owning to the influence of Typhoon Zola and the poor MSU resolutions, analysis showed that the 250-mb temperature field was not correctly calculated from the retrieved results, even though Zola had a wind speed of $100 \mathrm{kts}$. This suggested that large errors could be induced during temperature retrieval when the typhoon quickly intensifies into a solid structure. The 
Q/16 22:57-23:05 700mb(a.p)

0/19 06:01-06:09 700mb( s.p)
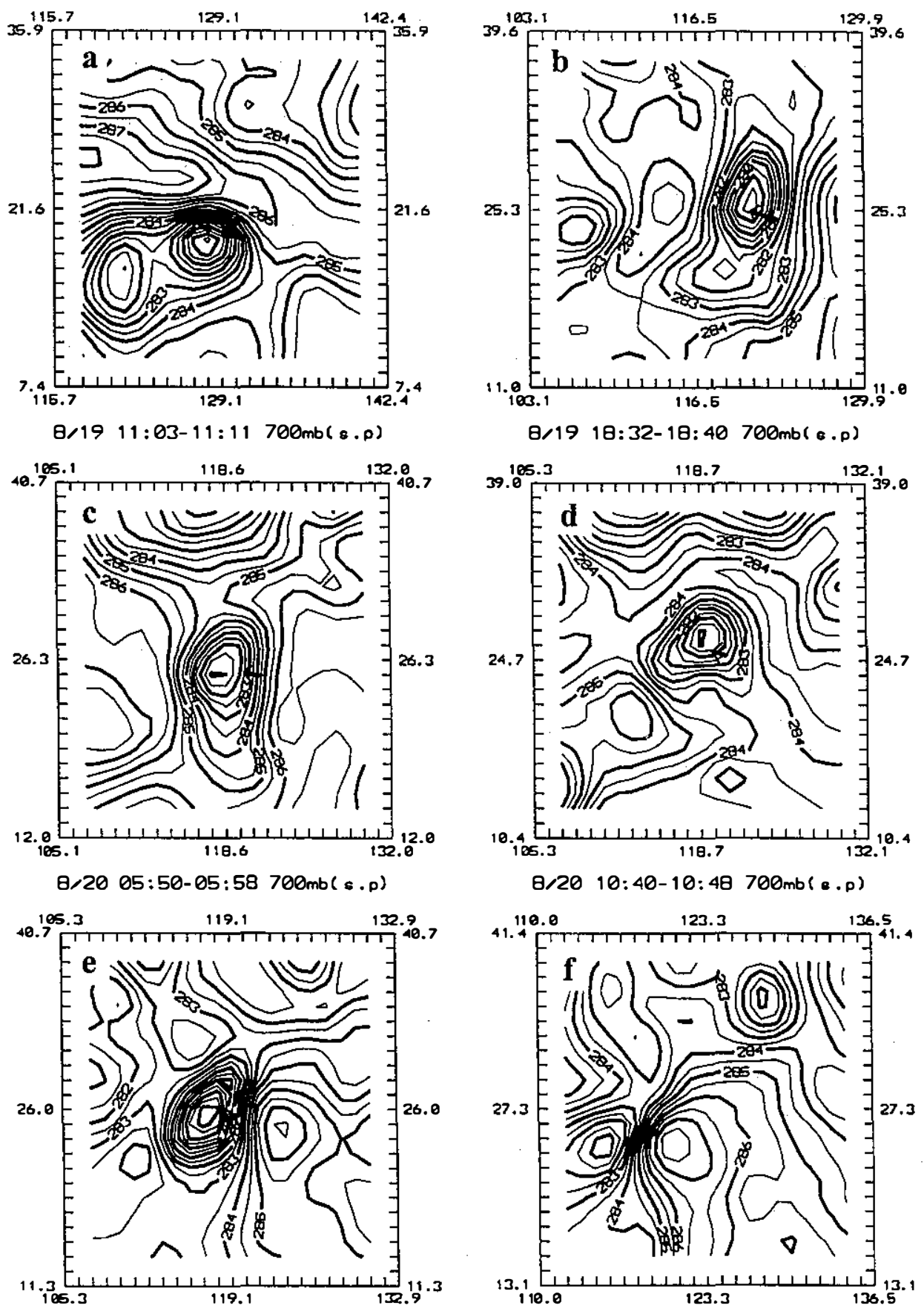

Fig. 2. Same as Fig. 1., but for the 700-mb temperature fields. 
Q/20 10:22-18:29 700mb( B.p)
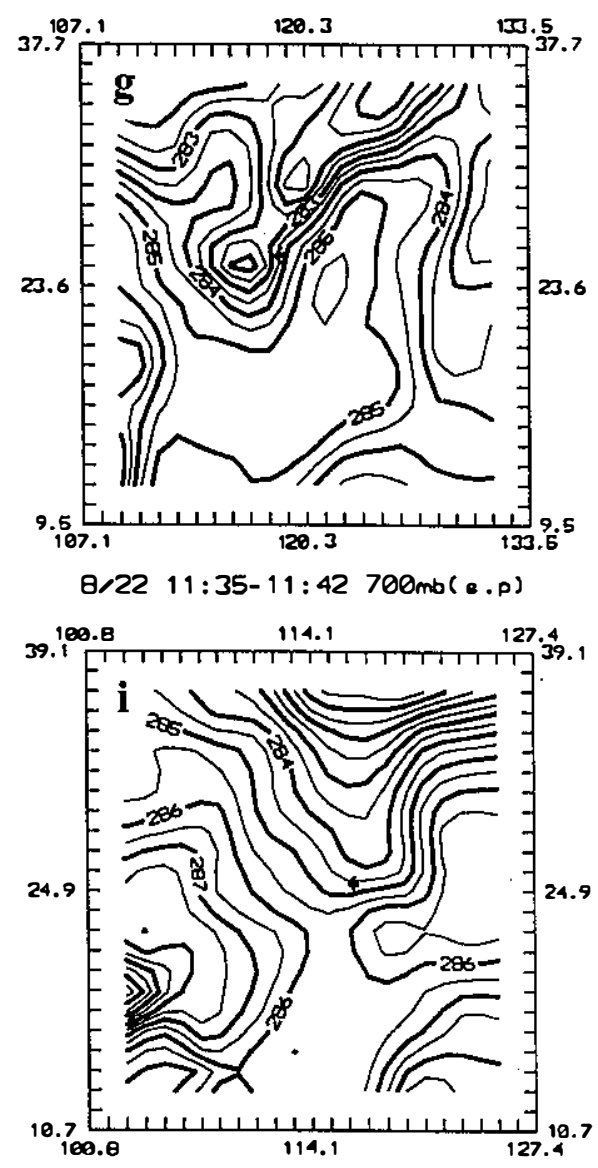

Q $8 / 21$ 10:10-18:18 700mb(s.p)

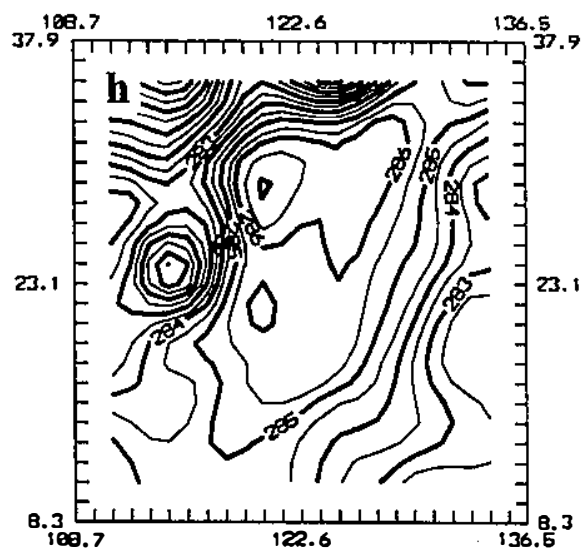

(Fig. 2. continued)

accuracy of the intensity estimation will certainly be significantly affected in such cases. On the other hand, the satellite observed warm-core location of Abe owned a maximum wind speed of $90 \mathrm{kts}$, which coincided with the aircraft observations because no other typhoon system was present nearby. And although Becky was influenced by Zola, its' warm-core was located at the storm's center because of its small size.

Based on the discussions in this study, the probable factors influencing the $250-\mathrm{mb}$ temperature fields are summarized as follows:

(1) The surface emissivity at MSU Channel 1 will be underestimated if the angle correction is not performed properly. This in turn causes an underestimation of the brightness temperature. In some weak typhoon cases, this may show the warm-core to appear separated from the typhoon's center location. In such situations, the retrieved warm-core structure will be asymmetrical.

(2) Because of the MSU's poor spatial resolution, the warm-core structure becomes asymmetrically obscured when two typhoon systems exist simultaneously in the same field-ofview. 
(3) Basically, the MSU data can not be used to estimate the gradient temperature inside various small typhoons that have a small eye region because of the poor resolution of the MSU data. Thus, the maximum wind speed can not be estimated by the small temperature anomaly that is related to the environment in some intensifying typhoon cases.

\subsection{0-mb Temperature Field}

From previous studies, the cold temperature anomaly can appear in the outer regions of the typhoon eye area below the $600-\mathrm{mb}$ height. This can be confirmed with the appearance of the cold-core structure in the 700-mb temperature field.

In the case of Typhoon Yancy, the center was located between a cold temperature anomaly to the west and a warm temperature anomaly to the east (Figs. 2e and 2f). Such particular warm-core patterns are mostly induced by the land surface. During the ITPP temperature retrieval process, MSU Channels 2, 3, and 4 were used. The corrected Channel 2 observation values would increase over the region of land, making atmospheric temperatures to be overestimated. This overestimation is more obvious in the $700-\mathrm{mb}$ temperature field, because its weighting function peak is roughly at the $700-\mathrm{mb}$ height. This warm-cold structure also occurred in Typhoon Winona when it moved near Mainland China.

In the early stages of Typhoon Zola where Yancy was located to the northwest, the coldcore appeared at the northwestern side of Zola's center. As stated earlier, owing to the poor MSU resolution of 110 kilometers, and the fact that the two systems were close together, the edges of the systems were not distinctly separated apart from the same MSU fields-of-view.

\section{TYPHOON INTENSITY AND TRACK MODELS}

Based on the discussion in section 2, we know that the wind speed at radius $r$ is proportional to the radial gradient of the brightness temperature at the same location. Because of the fact that the maximum wind speed in a typhoon generally appears around the outer typhoon eye area, we must know the values of the local temperature gradient in the typhoon eye region in order to estimate the maximum wind speed. Unfortunately, this local temperature gradient can not be retrieved by satellite data due to the poor spatial resolution of the MSU. Therefore, instead of using the local temperature gradient, this study assumed that the temperature difference between the warm-core and the environment would increase along with the temperature gradient inside the eye region. With such an assumption, the local temperature gradient may be replaced with the temperature anomaly.

\subsection{Single Variable Regression Algorithm}

In this study, 18 cases from Typhoon Yancy and Typhoon Abe were used to establish a typhoon intensity estimation model. Based on the previous discussion, differences in the typhoon center pressure, the environmental surface pressure, and the maximum wind speed may be related to the brightness temperature anomaly when the MSU channels were not affected by the surface emissivity. Among the MSU data, the Channel 3 owned the least influence from 
the surface emissivity. The height of its weighting function peak reached roughly to around the 250-mb layer where most typhoons' warm-cores are located generally. Therefore, the 250$\mathrm{mb}$ temperature anomaly was used as a predictor for estimating the maximum wind speed. Based on the 18 cases selected for this study, we found:

$$
\mathrm{V} \max =10.21 * \Delta \mathrm{T}_{250}+13.089
$$

where $V \max$ is the maximum wind speed (unit in kts), and $\Delta \mathrm{T}_{250}$ is the temperature anomaly (unit in $\mathrm{K}$ ) of 250-mb layer. The correlation equaled 0.58 and the mean maximum wind speed reached 66.4 kts (Table 1, Fig. 3a). This result shows the lower correlation could be observed in cases where stronger or weaker typhoon wind speeds are detected. In general, such results are not deemed satisfactory.

In the gradient wind equation, the square of the wind speed is a function of the radial temperature gradient. Therefore, we use the squared root of the $250-\mathrm{mb}$ temperature anomaly as a variable to find the maximum wind speed, which is written as:

$$
\mathrm{Vmax}=44.73 *\left(\Delta \mathrm{T}_{250}\right)^{0.5}-34.35
$$

The correlation equaled 0.62 , and the standard error was $13.7 \mathrm{kts}$. Obviously, some improvements can be witnessed in the result (Fig. 3b).

Generally, the maximum wind speed occurs at the $850-\mathrm{mb}$ height. The square root of the product of the $850-\mathrm{mb}$ cold-core temperature, $\mathrm{T}_{\mathrm{c} 850}$, and the $250-\mathrm{mb}$ temperature anomaly are used as a new variable, to acquire the wind speed regression relationship (Fig. 3c):

Table 1. Statistical comparisons of typhoon intensity estimations using different prediction models.

\begin{tabular}{llll}
\hline Model & R-Squared & STD(KtS) & RMSE \\
\hline$\triangle \mathrm{TB} 250$ & 0.58 & 14.6 & 18.1 \\
$(\triangle \mathrm{TB} 250)^{* *} 0.5$ & 0.62 & 13.7 & 21.0 \\
$(\triangle \mathrm{TB} 250 * \mathrm{~TB} 850)^{* *} 0.5$ & 0.62 & 13.8 & 18.1 \\
$\triangle \mathrm{TB} 250 \& \triangle \mathrm{TB} 700$ & 0.73 & 12.1 & 16.7 \\
{$[(\triangle \mathrm{TB} 250) \&(\triangle \mathrm{TB} 700)]^{* *} 0.5$} & 0.71 & 12.5 & 17.3 \\
$(\triangle \mathrm{TB} 250 * \mathrm{~TB} 850)^{* * 0.5} \&$ & 0.71 & 12.5 & 15.4 \\
$(\triangle \mathrm{TB} 700 * \mathrm{~TB} 850)^{* *} 0.5$ & & & \\
$\triangle \mathrm{TB} 250 \&(\triangle \mathrm{TB} 250)^{* * 2}$ & 0.65 & 13.7 & 19.3 \\
\hline
\end{tabular}




$$
V_{\max }=2.645 *\left(\left(\Delta \mathrm{T} 250 * \mathrm{~T}_{\mathrm{C850}}\right)^{0.5}\right)-34.783
$$

The correlation again equaled 0.62 , and the standard error was $13.8 \mathrm{kts}$, showing that the additional information provided by the $850-\mathrm{mb}$ temperature field further increased the accuracy than $\mathrm{Eq}(11)$.
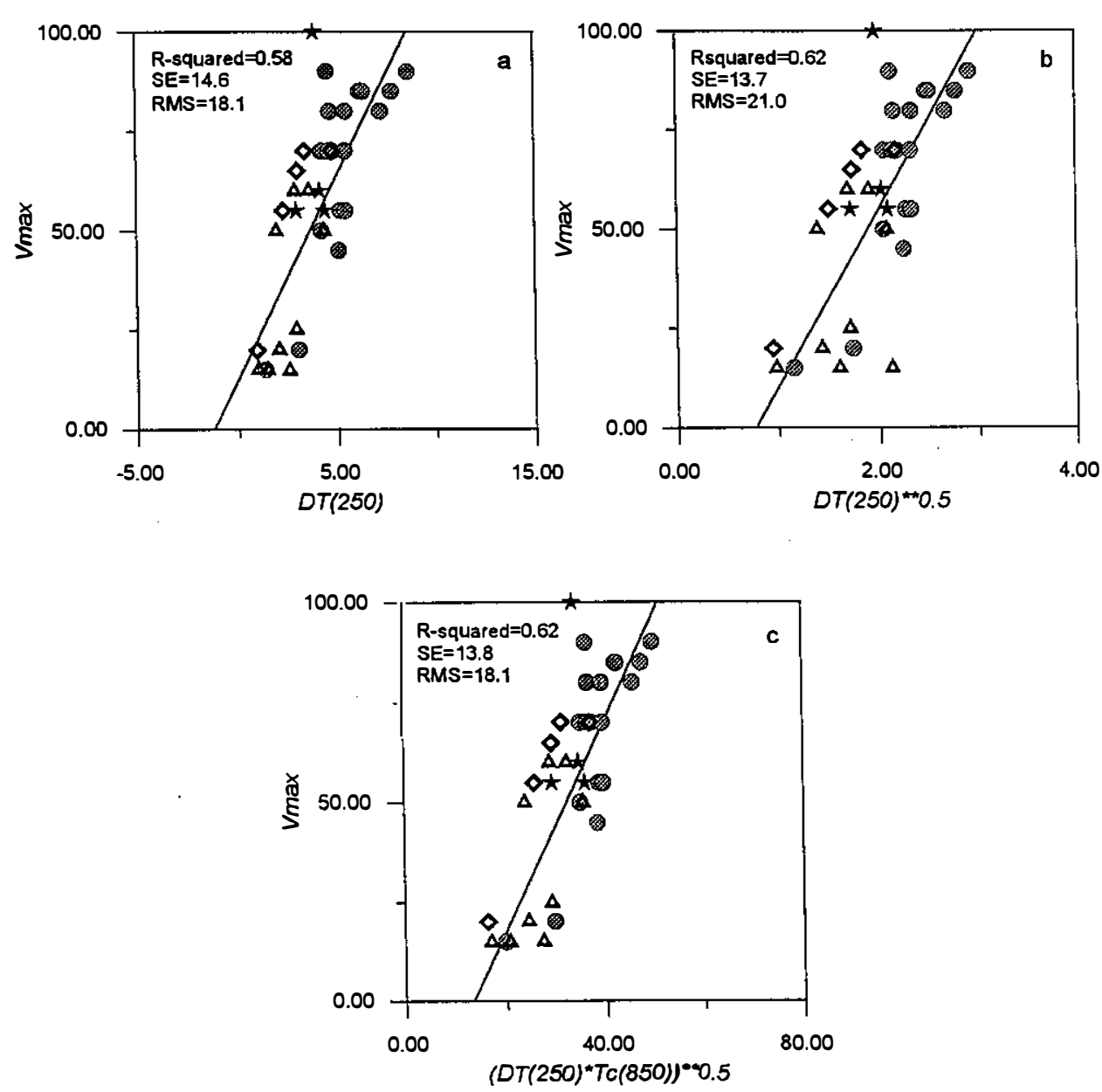

Fig. 3. The regression of typhoon intensity and check points estimated by using 250-mb temperature anomaly data. The circle dots show the regression points used for the estimation model. The triangle, square, and star symbols indicate the points for Typhoon Winona, Typhoon Becky and Typhoon Zola, respectively. The $\mathrm{x}$-axis of (a) is the 250 -mb temperature anomaly. The $x$-axis of (b) is the squared-root of the 250 -mb temperature anomaly. The $\mathrm{x}$-axis of (c) is the squared-root of the $250-\mathrm{mb}$ temperature anomaly times the squared-root of the $850-\mathrm{mb}$ cold-core temperature. The y-axis gives the typhoon's intensity. 


\subsection{Two Variables Regression}

In the single variable regression shown in section 5.1, we see that the input of the 250 -mb temperature anomaly owns the best correlation. In fact, a typhoon's thermal structure may sometimes vary very distinctly with different respective convective layers. For example, a warm-core structure existed in the 250-mb layer, but on the other hand, the opposite was witnessed in the 700 -mb height. Therefore, if information from the 250 -mb level is extracted only in estimating a typhoon's wind speed, the accuracy will drop a great deal. In this study, we suggested that the 700-mb temperature anomaly could also be pulled in to provide additional information for the wind speed estimation. The wind speed regression of two variables can be written as:

$$
\mathrm{Vmax}=5.793 * \Delta \mathrm{T}_{250}+4.443 * \Delta \mathrm{T}_{700}+32.828
$$

where $\Delta \mathrm{T}_{250}$ and $\Delta \mathrm{T}_{700}$ are the 250 and $700-\mathrm{mb}$ temperature anomalies, respectively. A total of 18 data sets from Typhoon Yancy and Typhoon Abe were analyzed.

Basically, Eq (14) is a three-dimensional line. For convenience of analysis, some transformations were performed to transform the three dimensional figures into two-dimensional ones. As we let $V \max$ to be plotted in the new $\mathrm{Y}$-axis, and let $\left(\Delta \mathrm{T}_{250}+4.443 * \Delta \mathrm{T}_{700} 15.793\right)$ to be plotted in the new X-axis, a new figure is obtained (Fig. 4a). The correlation equaled 0.73 and the standard error was at $12.1 \mathrm{kts}$, revealing that the 700 -mb information can significantly improve the estimation accuracy.

Similar to Fig. 3 b, we again use the root of the 700-mb temperature anomaly to be the second variable in establishing a new intensity model:

$$
\operatorname{Vmax}=28.573 *\left(\Delta \mathrm{T}_{250}\right)^{05}+6.729 *\left(\Delta \mathrm{T}_{700}\right)^{05}-1.624
$$

The results are shown in Fig. $4 \mathrm{~b}$, and the correlation and standard error equal 0.71 and $12.5 \mathrm{kts}$, respectively. The accuracy was worse than the result of Eq (14), but still proved to be better than Fig. 3b.

The root of the product of the $250-\mathrm{mb}$ and $700-\mathrm{mb}$ temperature anomalies and the square of the 850 -mb cold-core temperature are also used for,

$$
\mathrm{V} \max =1.682 *\left(\Delta \mathrm{T}_{250} * \mathrm{~T}_{\mathrm{c} 850}\right)^{0.5}+0.401 *\left(\Delta \mathrm{T}_{700} * \mathrm{~T}_{\mathrm{c} 850}\right)^{0.5}-1.63
$$

The results are shown in Fig. 4c. The correlation and standard error equal 0.71 and $12.5 \mathrm{kts}$, respectively. These results reveal that the usage of the 700-mb temperature anomalies can indeed improve the typhoon intensity estimation accuracy. The 250-mb temperature anomaly and its squared value were used in different cases of Typhoon Yancy and Typhoon Abe,

$$
\mathrm{Vmax}=24.462 * \Delta \mathrm{T}_{250}-1.388 *\left(\Delta \mathrm{T}_{250}\right)^{2}-19.843
$$


Liu et al.
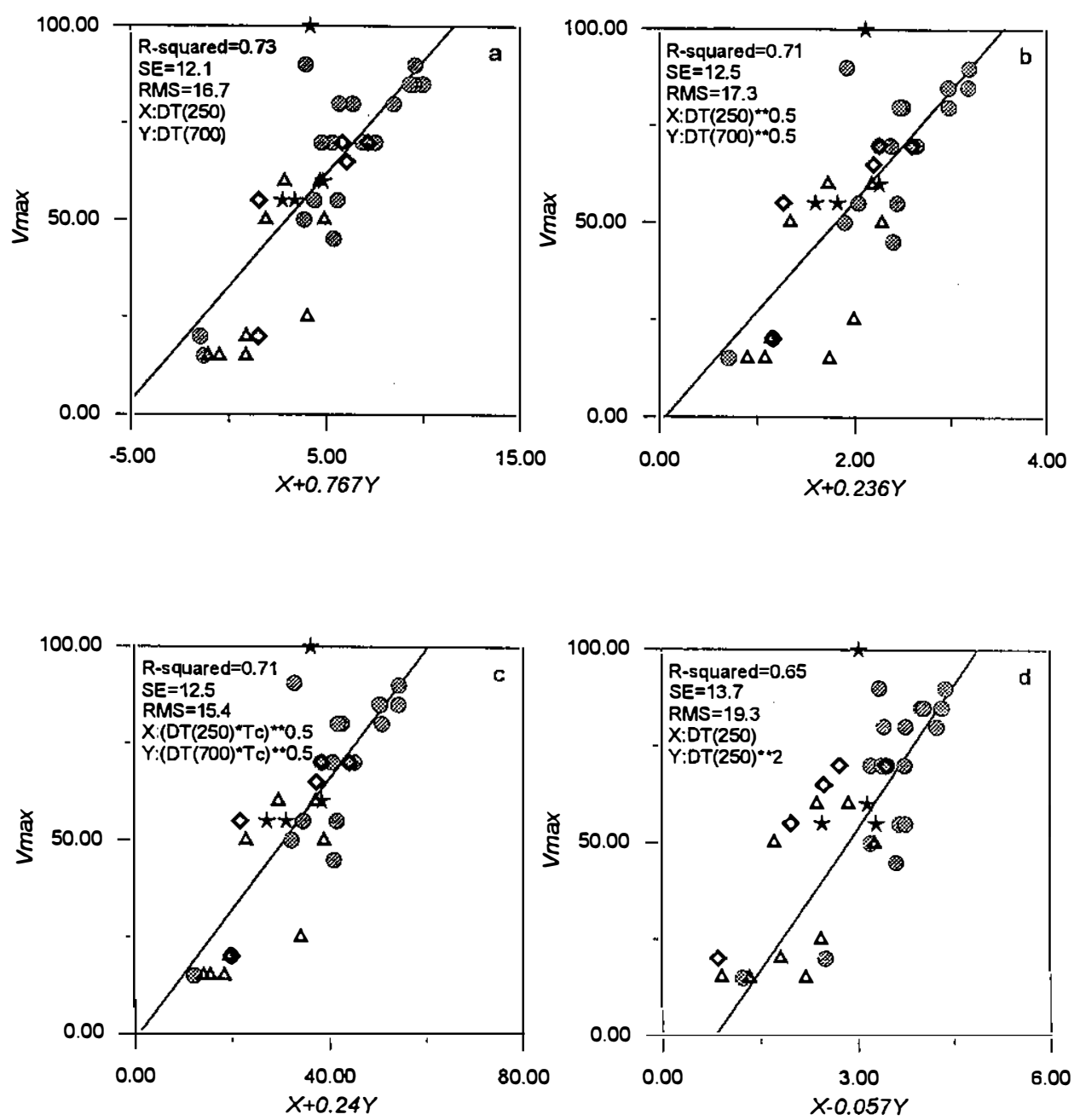

Fig. 4. Same as Fig. 3, but estimated by using the $250-\mathrm{mb}$ and $700-\mathrm{mb}$ temperature anomaly data. Figures. $4 \mathrm{a}, 4 \mathrm{~b}$ and $4 \mathrm{c}$ are the same as Figs. $3 \mathrm{a}, 3 \mathrm{~b}$ and $3 \mathrm{c}$, except when using the $700-\mathrm{mb}$ temperature anomaly data. Fig. $4 \mathrm{~d}$ used the 250-mb temperature anomaly data.

The results are shown in Fig. 4d, with the correlation and standard error being 0.65 and 13.7 kts, respectively. The accuracy of Eq (17) dropped significantly when the 700-mb temperature anomaly was not considered. This demonstrates that information from the 700-mb level serves as an important factor in the construction of an intensity estimation model.

\subsection{Accuracy and Error Analysis}

As discussed earlier, a total of 18 cases from Typhoon Winona, Typhoon Becky and Typhoon Zola were used to check the estimation accuracy. Figures. 3 and 4 illustrate the 
results of the $250-\mathrm{mb}$ and $700-\mathrm{mb}$ temperature anomaly model. Indeed, with the added 700 $\mathrm{mb}$ temperature anomaly information, it can be seen that the estimation accuracy is greatly improved.

From the figures, the root mean square for three typhoons is roughly between about 15 to $20 \mathrm{kts}$, and varied with the changes of the typhoons' intensities. Basically, the two-variable model had a better result than the single-variable model. Several cases that had a larger error could be witnessed, such as when Yancy's intensity reached 45 kts or when Abe's intensity was at $80 \mathrm{kts}$. These errors were induced by overestimated temperature retrievals caused by adjacent land field-of-views. The analysis study showed that the largest errors occurred in the Winona and Zola cases, especially when Winona's intensity dropped below $25 \mathrm{kts}$ and Zola's intensity was at it's strongest. When Winona grew weaker, the warm-core location shifted away from the typhoon center because of an overestimated temperature reading over the land. This difference may explain the reason for the inaccurate temperature anomaly. The error in the Zola cases during its stronger stages could have been induced by poor MSU resolution. Moreover, the temperature anomaly may not serve as a good indicator for a typhoon's intensity during periods of rapid intensification. This error grew significantly when the intensity surpassed 90 kts. Such situations can in fact also be found in Atlantic cyclones (Velden 1989), especially when their intensities surpass more than $100 \mathrm{kts}$.

\subsection{Typhoon Track Prediction}

As in the previous discussion, we know that the 700-mb cold-core structure can be found near a typhoon center in most cases. Analysis showed that the typhoon track tend to keep to its original course over the next interval of 6 hours if the lower brightness temperature area, which is induced by strong rainfall activity, was within a $3^{\circ}$ latitude from the typhoon center in the 700-mb (see also Figs. 2a, b, d for Typhoon Yancy). Otherwise, the typhoon would move towards in the cold-core direction. (see also Figs. $2 \mathrm{e}, 2 \mathrm{f}$ and $2 \mathrm{~g}$ ). Therefore, typhoon track prediction models can be separated into two types -a cold-core mode or an original mode. The prediction models can be written as:

and $\quad \begin{array}{ll}y=0.795 x+18.172 \quad \text { (cold-core mode), } \\ y=0.935 x+10.534 \quad \text { (original mode), }\end{array}$

where $y$ is the angle relating the orientation of the current typhoon center versus the location 6 hours earlier. The angle is located in between the line connecting the two centers and the vertical line that intersects with the position of the typhoon's center 6 hours ago. It is defined as zero degrees to the north and increases in a clockwise direction. $\mathrm{x}$ is defined in relating the positions of the cold-core versus the typhoon center in a similar fashion where the typhoon center serves as the intersection point with the vertical lines.

The results of the cold-core model are shown in Fig. 5a. The x-axis is the predicted typhoon rack angle, while the $y$-axis is the actual rack angle. The correlation was 0.93 and the standard error in the direction angle was at 17.9 degrees. The correlation error and standard error in the directional angle for the original mode were 0.92 and 14.3 degrees, respectively. Overall, the two models both provided a satisfying typhoon track prediction.

In this study, by utilizing the previous method, we also tried to put together information 

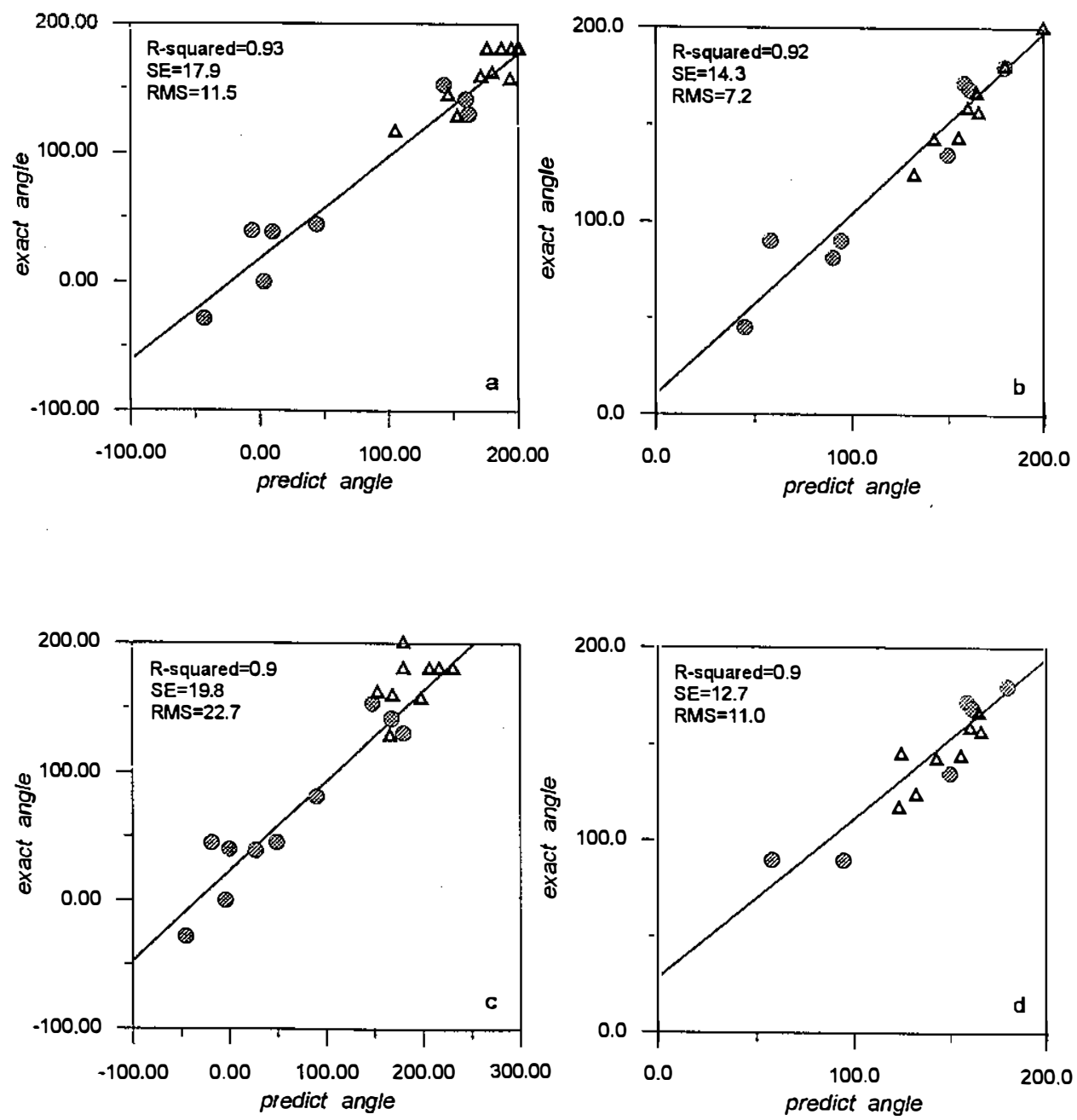

Fig. 5. (a)(b) The typhoon rack estimated by the 700 cold-core location data. (c) (d) The typhoon rack estimated by the $250-\mathrm{mb}$ warm-core location data. The (a)(c) cases show that the typhoon tracks move in the cold-core direction, and in the (b)(d) cases, the typhoon racks maintain their original path.

extracted from a 250-mb warm-core instead of an actual typhoon center, and the distance between a warm-core and a cold-core to predict the typhoon's path. Two prediction models were made:

and

$$
\begin{aligned}
& y=0.701 x+23.182 \quad \text { (cold-core mode), } \\
& y=0.83 x+28.48 \quad \text { (original mode) }
\end{aligned}
$$

The results are shown in Figs. 5c and 5d. The correlation and standard error in the directional angle for the cold-core mode stood at 0.9 and 19.8 degrees, respectively, and at 0.9 and 12.7 degrees, respectively, for the original mode. The results from Eq (20) and (21) were obviously 
Table 2. Statistical comparisons of typhoon track estimation using different prediction methods.

\begin{tabular}{llll}
\hline Model & R-Squared & STD(degree) & RMSE \\
\hline 700-mb cold-core Track & 0.93 & 17.9 & 11.5 \\
Track over the next 6 hours & 0.92 & 14.3 & 7.2 \\
250-mb warm-core and 250-mb & 0.90 & 19.8 & 22.7 \\
cold-core angle direction & & & \\
Track over the next 6 hours & 0.90 & 12.7 & 11.0 \\
\hline
\end{tabular}

worse than the ones obtained from Eq (18) and (19) (see also Table 2). The probable reason may be attributed to land effects or the presence of other typhoon or low pressure systems nearby.

\section{CONCLUSIONS}

This paper demonstrated that the use of satellite passive microwave data can help improve our understanding of a typhoon's characteristics. Based on our 250 and $700-\mathrm{mb}$ temperature field analyses, ITPP retrieval can be overestimated if MSU Channel 1 is not processed for angle correction. This will cause the warm-core location to be misplaced from the actual typhoon center to appear as a non-symmetrical pattern when the typhoon's intensity is small, or if land is located nearby.

The poor spatial resolution of the MSU fields may lead to limitations in the estimation of the maximum wind speed. Theoretically, the maximum wind speed has a single relationship with the radial temperature gradient within the typhoon's eye, but the poor resolution will limit the retrieval of the typhoon's eye temperature. Therefore, when the temperature anomaly in the outer area of a typhoon's eye is used instead of the typhoon eye itself to estimate the intensity, it may lead to inaccurate results, especially when the typhoon is intensifying.

The result will not be precise enough if only the $250-\mathrm{mb}$ temperature anomaly is considered in estimating the typhoon intensity. Fortunately, the problem can be greatly alleviated when the 700-mb temperature anomaly is added. Although the 700-mb cold-core has a totally different structure compared to the $250-\mathrm{mb}$ warm-core, this study suggests that the simultaneous usage of the information from these two layers achieves a better result. Furthermore, our results also show that the cold-core at the 700-mb could be a good indicator in the typhoon track prediction. Typhoons tend to stay to their original paths when their cold-core location overlays the warm-core. On the other hand, it tends to veer toward the cold-core direction when its cold-core is located behind the typhoon center. However, more cases should be further examined to confirm this behavior and establish a more rigorous statistical model.

As seen in the previous discussion, the angle correction process can not be made for MSU 
Channel 1 because of the lack of surface emissivity information. The results show that this limitation could induce a non-neglected estimation error. Hence, further studies in the angle correlation of the MSU Channel 1 are necessary to improve the typhoon intensity estimation. Meanwhile, establishing a rainfall model for the Taiwan area, and correcting the rainfall effects in ITPP retrieval is another major step to work forward to in acquiring a more accurate typhoon heat structure and temperature field. More importantly, as the new AMSU (Advanced Microwave Sensor) sensors are able to provide more channels and display sharper resolutions, $50 \mathrm{~km}$ in nadir, it should have a strong potential for typhoon intensity and track monitoring. Hence, the application of the superior AMSU data in typhoon studies is one of our next important study topics.

Acknowledgments This research was supported by the National Science Council under contracts NSC 87-2621-P-008-005 and NSC88-2625-Z-008-007. The authors want to thank Mr. Charlie C. K. Liang for his kindness in reviewing the English writing and editing.

\section{REFERENCES}

Allison L. J., E. B. Rodger, T. T. Wilheit, and R. W. Fett, 1974: Tropical cyclone rainfall as measured by the Nimbus 5 electrically scanning microwave radiometer. Bull. Amer. Meteor. Sec., 55, 1074-1089.

Anthes R. A., 1982: Tropical cyclones their evolution, structure and effects. Meteorological Monographs. Amer. Meteor. Soc., 19, 27-32.

Chang, S.W., A. J. Randall J., S. Raman, and J.-J. Shi, 1993: SSM/I observations of ERICA IOP 4, marine cyclone: A comparison with in situ observations and model simulation. Mon. Wea. Rev.,121, 2452-2464.

Chen W. J., and G. R. Liu, 1996: Using AVHRR data derived cloud parameters in TOVS retrievals. Proceeding of National Science Council, 20, 237-248.

Grody, N., 1983: Severe storm observations using the microwave sounding unit. J. Clim. Appl. Meteor., 22, 609-625.

Holton J. R., 1972: An introduction to dynamic meteorology. Academic Press, New York, pp 319.

Huang, H. J., G. R. Liu, and T. H. Lin, 1992: Western Pacific moisture analysis as observed from DMSP SSM/I measurements. TAO, 3, 233-265.

Kidder, S. Q., W. M. Gray, and T. H. Vonder Harr, 1980: Tropical cyclone surface pressure and winds from satellite microwave data. Mon. Wea. Rev., 108, 144-152.

Liu G. R., W. L. Smith, and T. H. Achtor, 1988: The use of VISSR data in VAS temperature soundings. J. Applied Meteorology, 27, 1309-1321.

Petty, B. W., and D. K. Miller, 1994: Satellite microwave observation of precipitation correlated with intensification rate in extratropical oceanic cyclones. Mon. Wea. Rev.,123, 1904-1911.

Rodgers, E. B., S. W. Chang, and H. F. Pierce, 1993: A satellite observational and numerical study of precipitation characteristics in western north Atlantic tropical cyclones. J. Appl. Meteor., 33, 129-139. 
Velden, C. S., and W. L. Smith, 1983: Monitoring tropical cyclone evolution with NOAA satellite microwave observation. J. Climate Appl. Meteor., 22, 714-724.

Velden, C. S.,1989: Observational analysis of North Atlantic tropical cyclones from NOAA polar-orbiting satellite microwave data. J. Appl. Meteor., 28, 59-70.

Velden, C. S., B. M. Goodman, and R. T. Merrill, 1991: Western North Pacific tropical cyclone intensity estimation from NOAA polar-orbiting satellite microwave data. Mon. Wea. Rev., 119, 159-168. 